\title{
Falls Injuries and Anxiety among Community-Dwelling Older Adults in the Midst of COVID-19 Lockdown Restrictions: Are these Helpful or Harmful?
}

\author{
Ray Marks \\ Department of Health and Behavior Studies, Columbia University, Teachers College, New York, NY 10027, USA \\ *Corresponding Author: Dr. Ray Marks, Department of Health and Behavior Studies, Teachers College, Columbia \\ University, Box 114, 525W, 120th Street, New York, NY 10027, United States.
}

\begin{abstract}
Falls among community dwelling older adults, which have been found to produce many adverse health outcomes have been studied for many years. Among the multiple potentially remediable falls risk factors identified in this body of literature is the presence of various anxiety states and symptoms. This review explores the possible link between excess anxiety and the risk for falling in the context of the home, during the prevailing coronavirus COVID-19 pandemic lockdowns, in particular. Drawn largely from the English language peer reviewed works posted on PUBMED and SCIENCE DIRECT largely between January 1, 2015-April 30, 2021), articles that focused on the topic of anxiety among older adults in community settings at the time of the COVID-19 pandemic were sought and examined. Although very limited in quantity, these data show, a sizable proportion of older adults confined to their homes continue to incur injurious falls at substantive rates. Moreover, these data may be underestimates, because only injurious falls have been closely studied over this time period. Based on past research showing those experiencing any form of anxiety are likely to be at higher risk for falling once or multiple times than non-anxious older adults and the fact COVID-19 produces high anxiety responses, it is concluded that more attention to this correlate alongside efforts to prevent COVID-19 infections are strongly indicated. More focused efforts to avert falls in the home based on current pandemic findings are also essential to consider in any future public health advisory on limiting mobility and access to life affirming resources to save lives.
\end{abstract}

Keywords: Aging; Anxiety, Community, COVID-19, Falls, Home-Isolation, Injuries, Older Adults, Prevention

\section{INTRODUCTION}

Falls and falls injuries, which represent an event resulting in the individual coming to rest inadvertently on the ground or floor or other lower level surface continue to prevail at high rates among communitydwelling adults 60 years of age or older, regardless of years of study and multifaceted preventive intervention attempts [1]. Commonly, producing marked long term progressive deficits in life quality, as well as tremendous financial and social costs among all aging societies $[2,3]$, the health of seniors who fall may be expected to decline and spiral downwards if action to prevent this is not forthcoming. In addition to fostering greater morbidity and mortality risks among older adults when compared to age-matched non fallers, falls may in fact prove deadly, hastening premature death [3]. In addition, falls leading to decreased social contacts in their own right, longterm physical disability, severe dependency and hospitalizations [2], are also strong predictors of life threatening fractures among the elderly [4].

As reported by White et al. [5], while psychological factors can undoubtedly heighten falls risk and falls consequences, there are also important psychological consequences of falls incurred by older adults, even those who were previously healthy, including depression, anxiety when walking or weight bearing, as well as generalized anxiety, activity related restrictions, and fear of falling, as stressed by Gonthier [3]. Fear of falling in addition, said to affect 20-43 percent of community-dwelling older adults, even if this fear is not attributable to an actual fall, commonly 
Falls Injuries and Anxiety among Community-Dwelling Older Adults in the Midst of COVID-19 Lockdown Restrictions: Are these Helpful or Harmful?

results in the individual restricting their physical and everyday activities, which can lead to functional declines, distress, sleep deprivation, depression, increased falls risk, and reduced life quality $[4,6]$, and a state where COVID-19 risk is likely to be heightened rather than abated.

Accordingly, the onset of the COVID-19 pandemic in December 2019, which itself was shown to impact seniors' wellbeing in particular, and that was found to generate much anxiety in its own right, would be expected to raise both falls risk as well as fears of falling during the ensuing socially restrictive stay-athome imperatives, while in turn, raising the risk for possible infection. Indeed, the associated withdrawal of home services and others designed to protect the elderly from infection exposure, regardless of their ability to function independently without detriment to their wellbeing, plus expecting them to carry out recommended home safety checks and desired home safety alterations independently in the face of needed social support and possible fears of moving seems very shortsighted at best. As well, possible weakness due to mild or moderate COVID-symptoms, frailty increases due to isolation effects, poor nutrition, reduced overall support, excess bone and muscle mass losses, declining balance and coordination abilities, and increased disability, could arguably be expected to further prevent any decline in the rates of injurious falls incurred in the homes of older adults over any pandemic restrictive period, despite injury declines in other spheres, for example sports injuries or motor vehicle injuries [7]. In fact, even more serious post pandemic fall injuries and consequences can surely be anticipated if anxiety states and others such as fear of moving remain unaddressed and of secondary or no overt preventive importance.

Unsurprisingly, as observed by Chiba et al. [8], despite a decline in most trauma related injuries during the recent COVID-19 pandemic that began in December 2019 , a notable $32 \%$ increase in the degree of ground level falls among the elderly group in particular was recently recorded in their trauma unit. Another current 2021 report has likewise shown that there has been an increase in home injuries over the pandemic period, and that falls remained the dominant mechanism of injury in 2020 in this regard, contributing 39.9\% of all hospitalizations according to Christey et al. [9].
Ruiz-Medina et al. [10] too reported falls rates to have experienced a significant increase during the lockdown period (18.9\% vs. $26.7 \%$; p = 0.026).

In addition, even if all home based falls that have occurred since the December 2019 COVID-19 pandemic onset have not been recorded or studied, Hazra et al. [11] found that while trauma related injuries as a whole decreased, an absolute increase in the number of falls on level ground $(+95.7 \%)$ was noted during the lockdown period. This was a finding similar to that of MacDonald et al. [12] and Rozenfeld et al. [13] who reported that many more injuries were sustained in the home during the COVID-19 outbreak, even though the proportions of injuries in all other localities decreased significantly. Injuries sustained during the COVID-19 outbreak were also more severe, specifically due to an increase in severe falls injuries.

High energy falls rates have similarly increased over the pandemic period [14], and symptoms such as falls observed in the older adults have been found to be associated with COVID-19 infections in the elderly [15]. Hashmi et al. [16] also found low energy falls in the home to be the most common mode of incurring trauma during the pandemic period, a finding supported by Gielen et al. [17].

Although not explicitly related to anxiety, it has also been shown in an observational study originating from a major United Kingdom trauma centre of patients admitted during a 10 -week period starting in March 2020 who were compared to a historical cohort of patients admitted during a similar time period in 2019 that this current cohort was older and more frail with more comorbidities and an increased falls rate. There was also a twofold increase in the risk of mortality in the 2020 cohort, which was explained by injury severity and frailty, when all other variables were controlled [18].

In another recent study, $\mathrm{Bu}$ et al. [19] who aimed to investigate the longitudinal association between loneliness, social isolation and falls amongst older adults in England, looking at both self-reported falls and falls that require hospital admissions found there was a $5 \%$ increase in the hazard of self-reported falls relative to one point increase in loneliness independent of socio-demographic factors. Both living alone and low social contact were associated with a greater hazard for self-reported falls even after 
controlling for socio-demographic factors, health and life-style differences. Similar results were also found for hospital admissions following a fall. The attributes that appeared impacted by isolation were both social contacts, as well as instrumental resources, both of which may be diminished in COVID-19 lockdown measures.

Other specific factors cited were: environment risks, for example tripping on a loose carpet, biomedical related risks, such as dizziness, degree of mobility, functional disability, vision, depression and physical inactivity.

In short, among the many factors that may mediate poor health, as well as heightened infection risk, psychological conditions, such as anxiety, which may occur in different forms, may conceivably be excessively heightened in response to unrelenting COVID-19 pandemic rulings and fears. A situation that can potentially predispose the older community bound adult to a heightened falls risk, very few efforts appear to be directed towards preventing this negative health cycle, even though available data reveal that the associated periods of home confinement in the context of COVID-19 has greatly increased the risk of mental ill-health [20], especially in terms of anxiety, which may be considerably elevated in older adults in response to the pandemic and its socioeconomic and health services ramifications [21-23], especially where lockdown rulings are prolonged or unpredictable.

\section{AIM}

In light of the aforementioned findings, and the concurrent observation that psychological symptoms such as anxiety, a very common psychological syndrome occurring in the older adult population either as either a generalized or specific chronic condition, as well as in response to a variety of stressful negatively perceived situations, this review strove to specifically examine what has been reported as regards the role of anxiety in falls-related risk among older community dwelling adults who may be confined and isolated for protracted periods in response to public health COVID19 preventive rulings. It also aimed to examine if more needs to be done to offset falls risk and by analogy infection risk, during future lockdowns that have been discussed by public health experts and policy makers, and if so, in what respect.

Based on what is known, it was hypothesized that anxiety, in its various forms, and that may predate COVID-19 or prevail as a reactive responses to this, or both, may be considerably heightened in the case of those older community dwelling adults, who are having to self-isolate amidst other resource lockdowns that they commonly access or employ, hence more likely to be distracted or fearful and incur falls at higher rates than are normally anticipated or evidenced [6]. It was also believed that basic falls fears among the elderly, as well as excess fears of falling even in response to non injurious home falls experienced during periods of imposed isolation and distancing, could provoke further anxiety, as well as an excess fear of falling that commonly leads quite rapidly to fear of movement, weakness, frailty, and ultimately possible fatal health implications as outline in Figure 1.

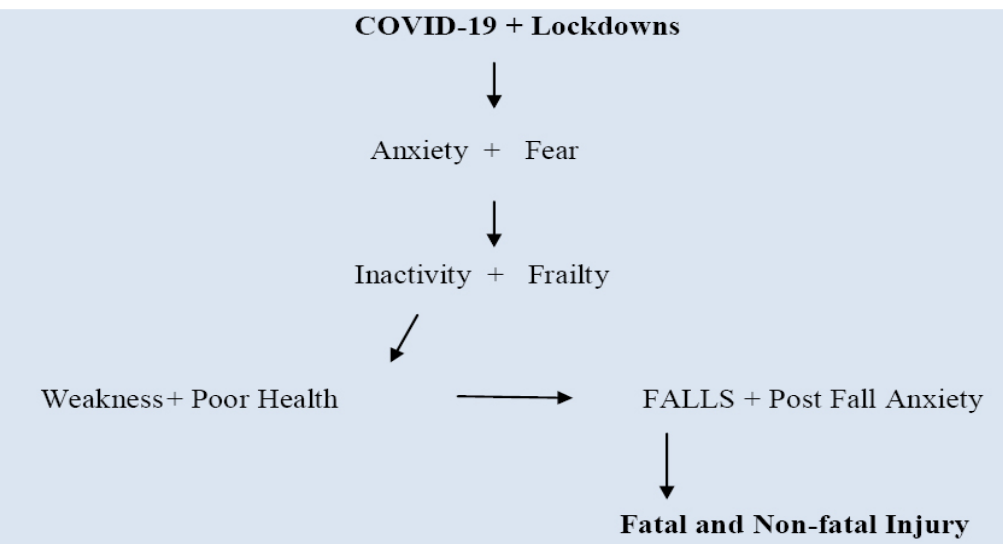

Adapted from [references: 1, 6, 7, 24-26]

Figure1. Hypothetical Interactions of COVID-19 Social Isolation Restrictions and Falls Risk and Anxiety as Applied to Older Community Living Adults 
Falls Injuries and Anxiety among Community-Dwelling Older Adults in the Midst of COVID-19 Lockdown Restrictions: Are these Helpful or Harmful?

\section{METHODS}

An extensive internet, plus PUBMED and SCIENCE DIRECT data base search was carried out over several days in order to locate recent articles or information sources detailing or examining falls risk and features of anxiety as related to COVID-19 and falling among older adults living in the community as published between 2015-2021. These databases were specifically selected owing to their being among the world's largest medical peer reviewed data sources and repositories and that are deemed reliable and representative of the documents reporting the issue at hand. The focus of the search was on falls among older adults, specifically those living independently in the community relative to the COVID-19 pandemic and its related forms of lockdowns. Limited largely to a five year search period commencing in April-2021, the key words applied were: anxiety, coronavirus, COVID19, falls, lockdowns, and older adults. All forms of relevant documentation were deemed acceptable, and after carefully scanning the most salient documents, the most relevant were downloaded and reviewed in more detail. A final search was conducted, and the information of interest was extracted and relayed in narrative form to include general background information, specific topical information, and the implications thereof. Nursing home studies, studies on younger or middle aged adults, studies on specific health conditions, and studies published before 2014 were largely excluded.

\section{RESULTS}

Although falls are common among older adults, the population most impacted by COVID-19, very few current publications address this topic specifically, especially from an anxiety perspective. However, even though Williams et al. [27] noted no significant association between anxiety disorders and falls among prior fallers who were depressed in their study, as observed in a systematic review by Payette et al [28] and Almeida et al. [18] who studied 41,098 community dwelling men and women, mean ages 70.0 \pm 8.9 years, a falls prevalence of $8.2 \%$ that was noted was higher among those who were older, female, and frail. As well, those exhibiting evidence of multiple medication usage and/or fear of falling, plus those with falls related psychological concerns were more likely to fall than those who did not take multiple medications or have any falls associated fears.

Ametaanalysis by Hallford etal.[29] too showed clinical anxiety and falls risk are indeed correlated, although the causal pathways are not clearly researched or understood. Holloway et al. [30] confirmed that falls in the home, common among community dwelling older adults, especially during COVID-19 isolation situations, may prove highly injurious [31], and that anxiety may mediate this association between falls and aging, especially among men.

In another study, where Fabrega-Cuadros et al. [32] assessed the impact of psychological distress and sleep quality on balance confidence, muscle strength, and functional balance in 304 community-dwelling middle-aged and older adults, the results of their multivariate linear and logistic regressions showed higher anxiety values, fatigue, and using sleep medication were linked to falling. Anxious older adults were also found to exhibit less proficiency on standing at an elevated level and undergoing postural sway tests than non anxious control subjects [33].

According to Serrano-Checa et al. [34] in addition to well known falls risk factors such as gait, dynamic balance, and functional mobility problems, sleep disturbances, anxiety, and depression are prevalent factors among older women. In particular, their study showed that poor sleep efficiency and the use of sleeping medication were related to decreased gait speed, poor functional mobility, which was linked to depression and the use of sleeping medication. Additionally, increased symptoms of anxiety and depression were associated with worsened dynamic balance. Unaddressed risk factors for falls, that may not only lead to more movement restraint than is desirable also include obesity [35], a fear of falling, post-fall anxiety syndrome, depression and anxiety [24], and excess frailty [7].

Ellmers et al. [36] who examined the relationship of anxiety and high-risk patterns of visual search in 44 older adults during adaptive locomotion among older adults deemed to be at a high risk of falling found a link between heightened fall-related anxiety and "high-risk" visual search behaviors to be associated with greater stepping errors. As per Viaje et al. [37] who investigated the effect of a visuospatial dualtask on stepping performance in older people with 
Falls Injuries and Anxiety among Community-Dwelling Older Adults in the Midst of COVID-19 Lockdown Restrictions: Are these Helpful or Harmful?

and without concern about falling and the impact of repeating this task in those with high concern about falling concluded that that people with higher general concern about falling experienced more difficulties during a dual-task condition than those with lower concern levels. Of further interest was that 'worse' sensorimotor and cognitive functioning heightened this effect.

Another highly relevant cross-sectional, as well as longitudinal study conducted by Choi et al. [38] that examined whether worrying about falls restricts social engagement in older adults showed anxiety was significantly associated with both informal and formal social engagement restriction at time two, even when controlling for falls incidents and changes in health status and other covariates. The findings underscored the importance of reducing fall worry and preventing social disengagement in late life, as well as possibly during any ensuing period of imposed or prolonged home-based social isolation.

As observed by Zhao et al. [39], whereas falls in community-dwelling older adults are a complicated phenomenon attributed to a variety of socio demographic factors, health conditions, functional problems, and environmental factors, this group found that homebound or semi-homebound older adults were $50 \%$ more likely to experience a fall than non-homebound individuals. Impaired balance was the strongest falls predictor, followed by problems moving around in the home. Additional risk factors were arthritis, depression or anxiety.

Other reports imply that the negative impact of social isolation, confinement, and social distancing on mental and physical health of older adults, and their connection to falls vulnerability, as well as poor health cannot be underestimated $[19,21]$. In addition, a possible accompanying lack of outdoor exposure and resultant vitamin D deficiency may likewise contribute to an increased falls and fall injury risk through its effect on muscle, and bone health, in its own right [40]. Social isolation may also impact sleep quality, pain, and the ability to lead an active lifestyle in the presence of excess fear [41]. At the same time, excess drug and/or medication usage as an outcome of the intersection of these converging factors may explain the increases of injurious medication/drug overdose visits observed over the pandemic period by Harmon et al. [42] as well as falls [43, 44]. Sitdhiraksa et al. [45] found approximately $35 \%$ of previous older fallers 80 years of age or above who were living alone to also report fear of falling, even if they had not fallen and this was associated in part with anxiety, depression, and balance impairments. In another study of 102 COVID-19 older patients, mean age, 85 years, the most common atypical symptoms were confusion (29\%), difficulty walking (13\%), and falls (8\%). In-hospital and 30-day mortality rates were highest in patients with confusion or who had fallen [46].

Table1. Summary of Selected Key Study Findings Concerning Falls and Anxiety

\begin{tabular}{|l|l|}
\hline INVESTIGATORS & FINDING \\
\hline Ang et al. [24] & Elderly fallers/near fallers may develop post fall anxiety \\
\hline Downton et al. [47] & Older fallers have higher anxiety rates than non fallers \\
\hline Fabrega-Cuadros et al. [32] & Greater anxiety and sleep medication heighten falls risk \\
\hline Hallford et al. [29] & There is a link between anxiety and falling \\
\hline Holloway et al. [30] & There is an association between falling and anxiety in men \\
\hline Iaboni et al. [48] & Excess fear of falling and falls risk are related \\
\hline Serrana-Cherca et al. [37] & Sleep quality, anxiety, and depression correlate to falls \\
\hline Zhao et al. [49] & Anxiety predicts falls in community dwelling older adults \\
\hline
\end{tabular}

In short, as per Table 1, even with limited data and the fact that the precise number of falls attributable to anxiety factors related to COVID-19 confinement rules and fears are hard to consolidate, as many may not have been recorded overtly, COVID-19 based stay-at-home- impacts on social mobility, as well as resource access, plus a high presence of chronic health conditions and anxiety in face of the persistent and unanticipated lockdowns appears to produce or induce ongoing and cascading influences on factors known to enhance fear, 
Falls Injuries and Anxiety among Community-Dwelling Older Adults in the Midst of COVID-19 Lockdown Restrictions: Are these Helpful or Harmful?

anxiety, and with this falls vulnerability among older possibly immunocompromised and chronically ill community living adults [50] that warrants immediate and carefully considered public health interventions 24]. Indeed, without immediate and dedicated efforts, it is likely that persistent falls injuries, including more severe injuries than those presently being reported since the inception of the pandemic, will ensue, even though many other forms of injurious trauma have been found to decline. Alternately, unrecognized or unaddressed risk factors for falls among the older population may foster even more serious recurrent falls [24] and unwanted possibly pandemic mediated extremely adverse overall health outcomes. The added element of anxiety, in the face of fear-provoking media messages, and its overall impact on falls fears, as well as falls, may place many older adults trying to age 'in place' at an even greater risk of premature mortality from lack of insight in this regard, than possibly COVID-19 infections per se.

Based on past research, key prevention strategies that may need to be forthcoming are:

\begin{tabular}{ll}
\hline - & Education and educational materials \\
- & Adapted forms of exercise \\
- & Effective policies to reduce risk [1]. \\
\hline
\end{tabular}

Other strategies that may warrant resource allocation include:

$>$ Professional home safety assessments and possible modifications

$>$ The assessment and possible reduction of or withdrawal of psychotropic drugs

$>$ Multifactorial interventions that involve: individualized fall-risk assessments followed by tailored interventions and referrals to address identified risks

$>$ Vitamin D supplements for those who are vitamin D deficient or at risk

In addition a body of research supports non pharmacologic anxiety alleviating interventions, sleep based education, dietary and weight control strategies, face to face counseling, and requiring landlords to make necessary modifications to homes, plus the enforcement of building standards, as indicated [1].

\section{Discussion}

Falls have been and continue to be a leading cause of death among older persons in the United States and elsewhere, affecting at least one in three or as many as one in two elderly adults over age 65 annually. A further 20-30 percent of survivors will suffer moderate to severe injuries sufficient to reduce mobility and independence and about two-thirds of fallers may suffer another fall within the next six months that requires hospital admission. In addition to enormous physical costs in terms of disability, if the faller survives, the direct economic costs of falling injuries are enormous [24].

Unfortunately, current data reveal, no abatement of this problem, but if anything, an exacerbation of falls in the home that that are highly injurious requiring hospitalization as assessed in multiple sites over the COVID-19 pandemic period. In addition to the unavailability of many resources that normally keep older adults in their homes physically and mentally healthy to a high degree, their abrupt removal, limited substitution, and anxiety provoking situation of being asked to isolate and socially distance without due notice must surely be one of many contributing factors to these increases in injurious falls. Moreover, anxiety in general has been found to be a cause of falls in healthy older adults [51], while state anxiety in its various forms, coupled with reactive responses to COVID-19 stay at home rules in the form of anxiety must surely be implicated as well.

In this regard, if one examines current public health guidelines for protective older adults health status, these commonly predate COVID-19 or focus solely on COVID-19 prevention. However, those designed to address home falls prevention, may clearly not be tenable without specific external forms of intervention, even though evidence based. As well, virtual interactions with providers to replace in person counseling may not prove universally efficacious [21], especially in the case of delivering cognitive behavioral therapy to relieve anxiety [52]. In addition, withour help, appointments for timely cataract evaluations and operations, physical therapy interventions to enhance balance and strength, and primary care inputs to foster osteoporosis treatments [24] may similarly be extremely challenging to pursue in the context of extended lockdowns, travel restrictions, 
Falls Injuries and Anxiety among Community-Dwelling Older Adults in the Midst of COVID-19 Lockdown Restrictions: Are these Helpful or Harmful?

and service closures. At the same time, in the absence of support, fears of exercising alone, even if videos are available, as well as the possible presence of excess pain, impaired balance, and arthritic complications, may yet discourage, rather than encourage, highly recommended physical activity participation [40], as well as needed weight control [35].

Moreover, expecting older adults with mild cognitive impairments and/or physical impairments to be able to examine or implement home safety instructions, repair or eliminate unsafe hazards, as indicated in many home based falls prevention imperatives during lockdowns appears impossible to envision for those who live alone, and have lost their social support linkages as well as their vision, hand function, possible strength, and balance capacity. In fact, Welsh et al. [53] have shown some $31 \%$ of injuries were secondary to a new activity taken up during lockdown, implying that this may be a recommendation that should receive due consideration before advancing the idea.

Similarly asking house bound older adults to follow programs that focus on challenging balance and coordination exercises to enhance cognitions, and coordination in the absence of supervision in the home during lockdowns, and where older adults may already be weak, and vulnerable, must be challenged. These may not only be unsafe, and provoke falls, but may provoke undue anxiety and falls fears that predict falls, even if they were deemed effective in the pre pandemic states for anxious older individuals when conducted under supervision $[54,55]$.

Indeed, the widespread use of exercise as a falls prevention strategy as advocated by public health personnel and others must surely be carefully reviewed in the context of COVID-19 lockdowns, given the finding that while the number of trauma operations completed decreased by $38 \% ; 55 \%$ of injuries occurred inside the home and $44 \%$ outside the home during daily exercise [53].

According to Zhao et al. [39] who examined risk factors for falls in homebound community-dwelling older adults among 1,356 homebound community of dwelling older adults aged 65 and above, in absence of any policy directives to alleviate any preventable home based falls risk among vulnerable older adults, one can anticipate an immense increase in falls injuries in the future along with an increased burden of chronic health problems, plus emotional and functional health challenges and limitations. Those who exhibit anxiety and fear of falling, or have fallen recently or take multiple medications are most likely to be at high risk in this regard [56], unless timely insightful precautions against this possibility are taken.

Gill et al. [57] meantime, found their multi component intervention of no benefit on well being in the context of falls, and anxiety among older adults in pre pandemic times, suggesting more careful analysis of what is needed, and why needs to be forthcoming. Possible prevailing issues not discussed in the literature to any degree are how to foster exercise that is safe in the home, and tailored to the health status of the older adult who is suddenly unable to pursue their current exercise program in the community or under supervision, as well as risk factors related to over exercising, incorrect exercise approaches, for example among the frail. The role of diet and prescription medications in mediating falls risk in the absence of direct face to face counseling opportunities, which also warrants high attention, is not discussed to any degree. As well, the role of timely comprehensive home safety checks, balance, vision and hearing checks and how these should proceed in the face of service restrictions, has not been identified or specifically discussed in the context of current ongoing socially restrictive lockdowns. What older adults should do if they fall, either inside or outside the home while alone, is also not wisely communicated to those who may not have had time to prepare to prevent homes based falls and secure their personal safety, and have no link to the internet or apps. Moreover, even if helpful, group therapy for anxiety reduction, and other in-person anxiety reducing intervention strategies may be on hold or limited during the pandemic, even though these and other adaptive strategies to improve knowledge, awareness and self-coping are possibly vital in mitigating the risk of loneliness, anxiety and depression among isolated vulnerable community dwelling older adults that can lead to falls [21], as well as poor health [47]. As well, unrelieved or excess fears and anxiety can strongly predict the use of antianxiety medications that are known precursors of falls $[43,44]$, especially in the event the pandemic situation lasts for months or years [40].

Other data imply that attention to all fall risk factors in the homes of older community dwelling adults, 
Falls Injuries and Anxiety among Community-Dwelling Older Adults in the Midst of COVID-19 Lockdown Restrictions: Are these Helpful or Harmful?

such as a limited ability to safely retrieve, and carry delivered food packages and others, poor lighting, as well as other environmental hazards in their immediate surroundings, should not be neglected. At the same time, keeping older adults healthy and protected from succumbing to COVID-19 due to excess fear, anxiety, weakness or frailty [50] clearly warrants specific ongoing insightful attention. Identifying and limiting excess stress as far as possible, as well as muscle and bone mass losses through salient nutrient and supplements, plus practical stress control strategies such as relaxation [59], appears of equally high relevance here [7], as does the use of a 'falls risk scoring' measure [47]. Along with efforts to reduce or minimize anxiety and fears and promote psychological resilience [50], in contrast to fear provoking media messages and harsh rulings to avert the spread of mortal infections, perhaps a compassionate, caring, holistic approach to securing all elements of wellbeing, including emotional wellbeing, may render the best protection as regards home bound older adults health and safety, as well as that of their communities at large [60].

\section{ConCLUSIONS}

- Although vaccines may reduce the immediacy of death and severe COVID-19 infections, restrictive access and mobility as well as social strategies to mitigate COVID-19 that prevail and may prevail for some time to come, may continue to produce high rates of anxiety, and fear that predict fall severity, low energy as well as high fall injuries and recurrent falls among older home bound adults, along with a myriad of unwanted costly albeit potentially preventable health disabling effects.

- To avert a predictable cascade of falls events and their highly negative and detrimental costly consequences in current as well as post pandemic times and that may yet entail excess persistent anxiety symptoms, it appears reasonable to propose that more attention to promoting psychological health, accessibility to resources, and a high life quality, in addition to reducing infection risk among vulnerable older community based adults is warranted.

- Indeed, the negative link between falls, inactivity, social isolation, poor health, anxiety, excess frailty, and COVID-19 susceptibility and restrictions as these are found to affect older adults living in the community, not only warrants immediate attention in our view, but what efforts are of highest priority here, should be discussed and studied more intently and comprehensively and without delay by medical sociologists, health providers, psychologists, policy makers, researchers, and other health personnel in conjunction with community members and public health agencies and community organizations that serve the elderly.

\section{REFERENCES}

[1] World health Organization HO. Falls. Retrieved April 26, 2021 from: https://www.who.int/ news-room/fact-sheets/detail/falls

[2] Almada M, Brochado P, Portela D, Midão L, Costa E. Prevalence of falls and associated factors among community-dwelling older adults: a crosssectional study. Journal of Frailty and Aging. 2021;10(1):10-16. doi: 10.14283/jfa.2020.44.

[3] Gonthier R. Epidemiology, morbidity, mortality, cost to society and the individual, and main causes for falls. Bulletin de l'Académie Nationale de Médecine. . 2014;198(6):1025-1039. French.

[4] Yusuf AA, Hu Y, Chandler D, Crittenden DB, Barron RL. Predictors of imminent risk of fracture in Medicare-enrolled men and women. Archives of Osteoporosis. 2020;15(1):120. doi: 10.1007/ s11657-020-00784-7.

[5] White UE, Black AA, Wood JM, Delbaere K. Fear of falling in vision impairment. Optometry and Vision Science. 2015;92(6):730-735. doi: 10.1097/OPX.0000000000000596.

[6] Casagrande M, Favieri F, Tambelli R, Forte G. The enemy who sealed the world: effects quarantine due to the COVID-19 on sleep quality, anxiety, and psychological distress in the Italian population. Sleep Medicine. 2020;75:12-20.

[7] Briguglio M, Giorgino R, Dell'Osso B, Cesari M, Porta M, Lattanzio F, Banfi G, Peretti GM. Consequences for the elderly after COVID-19 isolation: FEaR (Frail Elderly amid Restrictions). Frontiers of Psychology. 2020;11:565052. doi: 10.3389/fpsyg.2020.565052. 
Falls Injuries and Anxiety among Community-Dwelling Older Adults in the Midst of COVID-19 Lockdown Restrictions: Are these Helpful or Harmful?

[8] Chiba H, Lewis M, Benjamin ER, Jakob DA, Liasidis P, Wong MD, Navarrete S, Carreon R, Demetriades D. "Safer at home": The effect of the COVID-19 lockdown on epidemiology, resource utilization, and outcomes at a large urban trauma center. Journal of Trauma and Acute Care Surgery. 2021;90(4):708-713. doi: 10.1097/ TA.0000000000003061.

[9] Christey G, Amey J, Singh N, Denize B, Campbell A. Admission to hospital for injury during COVID19 alert level restrictions. New Zealand Medical Journal. 2021;134(1531):50-58.

[10] Ruiz-Medina PE, Ramos-Meléndez EO, Cruz-De La Rosa KX, Arrieta-Alicea A, Guerrios-Rivera L, Nieves-Plaza M, Rodríguez-Ortiz P. The effect of the lockdown executive order during the COVID19 pandemic in recent trauma admissions in Puerto Rico. Injury Epidemiology. 2021;8(1):22. doi: 10.1186/s40621-021-00324-y.

[11] Hazra D, Jindal A,FernandesJP,Abhilash KP. Impact of the Lockdown due to COVID-19 Pandemic on the Spectrum and Outcome of Trauma in India. Indian Journal of Critical Care and Medicine. 2021;25(3):273-278. doi: 10.5005/jp-journals10071-23747.

[12] MacDonald DRW, Neilly DW, Davies PSE, Crome CR, Jamal B, Gill SL, Jariwala AC, Stevenson IM, Ashcroft GP; SCORE authors. Effects of the COVID-19 lockdown on orthopaedic trauma: a multicentre study across Scotland. Bone Joint Open. 2020;1(9):541-548. doi: 10.1302/26331462.19.BJO-2020-0114.R1.

[13] Rozenfeld M, Peleg K, Givon A, Bala M, Shaked G, Bahouth H, Bodas M. COVID-19 changed the injury patterns of hospitalized patients. Prehospital and Disaster Medicine. 2021 Mar 1:1-9. doi: 10.1017/S1049023X21000285.

[14] Lim MA, Mulyadi Ridia KG, Pranata R. Epidemiological pattern of orthopaedic fracture during the COVID-19 pandemic: a systematic review and meta-analysis. Journal of Clinical Orthopedics and Trauma. 2021 May;16:16-23. doi: 10.1016/j.jcot.2020.12.028.

[15] Gawronska K, Lorkowski J. Falls as one of the atypical presentations of
COVID-19 in older population. Geriatric Orthopedic Surgery and Rehabilitation. 2021 Feb 21;12:2151459321996619. doi: $10.1177 / 2151459321996619$.

[16] Hashmi PM, Zahid M, Ali A, Naqi H, Pidani AS, Hashmi AP, Noordin S. Change in the spectrum of orthopedic trauma: effects of COVID-19 pandemic in a developing nation during the upsurge; a cross-sectional study. Annals Medicine and Surgery (Lond). 2020;60:504-508. doi: 10.1016/j.amsu.2020.11.044.

[17] Gielen AC, Bachman G, Badaki-Makun O, Johnson RM, McDonald E, Omaki E, Pollack Porter KM, Ryan L, Shields W. National survey of home injuries during the time of COVID-19: who is at risk? Injury Epidemiology. 2020 Nov 11;7(1):63. doi: 10.1186/s40621-020-00291-w.

[18] Adiamah A, Thompson A, Lewis-Lloyd C, Dickson E, Blackburn L, Moody N, Gida S, La Valle A, Reilly JJ, Saunders J, Brooks A; ICON Trauma Study Group. The ICON Trauma Study: the impact of the COVID-19 lockdown on major trauma workload in the UK. European Journal of Trauma and Emergency Surgery. 2021 Feb 9:1-9. doi: 10.1007/s00068-020-01593-w.

[19] Bu F,Abell J,Zaninotto P, Fancourt D. Alongitudinal analysis of loneliness, social isolation and falls amongst older people in England. Science Report 2020;10:20064. ttps://doi.org/10.1038/ s41598-020-77104-z

[20] Jané-Llopis E, Anderson P, Segura L, Zabaleta E, Muñoz R, Ruiz G, Rehm J, Cabezas C, Colom J. Mental ill-health during COVID-19 confinement. BMC Psychiatry. 2021;21(1):194. doi: 10.1186/ s12888-021-03191-5.

[21] Robb CE, de Jager CA, Ahmadi-Abhari S, Giannakopoulou P, Udeh-Momoh C, McKeand J, Price G, Car J, Majeed A, Ward H, Middleton L. Associations of social isolation with anxiety and depression during the early COVID-19 pandemic: a survey of older adults in London, UK. Frontiers in Psychiatry. 2020 Sep 17;11:591120. doi: 10.3389/fpsyt.2020.591120.

[22] Wang ZH, Qi SG, Zhang H, Mao PX, He YL, Li J, Xiao SY, Peng HM, Sun WW, Guo HY, Liu M. [Impact 
Falls Injuries and Anxiety among Community-Dwelling Older Adults in the Midst of COVID-19 Lockdown Restrictions: Are these Helpful or Harmful?

of the COVID-19 epidemic on anxiety among the elderly in community]. Zhonghua Yi Xue Za Zhi.2020;100(40):3179-3185. Chinese. doi: 10.3760/cma.j.cn112137-20200720-02167.

[23] Cosco T, Fortuna K, Wister A, Riadi I, Wagner K, Sixsmith A. COVID-19, social isolation, and the mental health of older adults: a digital catch-22. Journal of Medicine and Internet Research. 2021 Apr 15. doi: 10.2196/21864.

[24] Ang GC, Low SL, How CH. Approach to falls among the elderly in the community. Singapore Medical Journal. 2020;61(3):116-121. doi: 10.11622/ smedj.2020029.

[25] Hayashi T, Umegaki H, Makino T, Huang CH, Inoue A, Shimada H, Kuzuya M. Combined impact of physical frailty and social isolation on rate of falls in older adults. The Journal Of Nutrition, Health \& Aging. 2020;24(3):312-318.

[26] Pohl JS, Cochrane BB, Schepp KG, Woods NF. Falls and social isolation of older adults in the National Health and Aging Trends Study. Research in Gerontological Nursing. 2018;11(2):61-70.

[27] Williams, L.J., Pasco, J.A., Stuart, A.L. et al.Psychiatric disorders, psychotropicmedication use and falls among women: an observational study. BMC Psychiatry. 2015;15:75. https://doi. org/10.1186/s12888-015-0439-4

[28] Payette M-C, Bélanger C, Léveillé V, Grenier S. Fall-related psychological concerns and anxiety among community-dwelling older adults: systematic review and meta-analysis. PLoS ONE 2016;11(4):e0152848. https://doi. org/10.1371/journal.pone.0152848

[29] Hallford, DJ, Nicholson G, Sanders K, McCab, MP. The association between anxiety and falls: a meta-analysis. Journals of Gerontology Series B: Psychological Sciences and Social Sciences. 2017;72(5);729-741.

[30] Holloway KL, Williams LJ, Brennan-Olsen SL, Morse AG, Kotowicz MA, Nicholson GC, Pasco JA. Anxiety disorders and falls among older adults. Journal of Affective Disorders, 2016;205:20-27.

[31] Zhu Y, Chen W, Xin X, Yin Y, Hu J, Lv H, Li W, Deng $X$, Zhu C, Zhu J, Zhang J. Epidemiologic characteristics of traumatic fractures in elderly patients during the outbreak of coronavirus disease 2019 in China. International Orthopaedics. 2020;44(8):1565-1570.

[32] Fábrega-Cuadros R, Aibar-Almazán A, MartínezAmat A, Hita-Contreras F. Impact of psychological distress and sleep quality on balance confidence, muscle strength, and functional balance in community-dwelling middle-aged and older people. Journal of Clinical Medicine. 2020 Sep 22;9(9):3059. doi: 10.3390/jcm9093059.

[33] Sturnieks DL, Delbaere K, Brodie MA, Lord SR. The influence of age, anxiety and concern about falling on postural sway when standing at an elevated level. Human Movement Science. 2016 Oct;49:206-15. doi: 10.1016/j. humov.2016.06.014..

[34] Serrano-Checa R, Hita-Contreras F, JiménezGarcía JD, Achalandabaso-Ochoa A, AibarAlmazán A, Martínez-Amat A. Sleep quality, anxiety, and depression are associated with fall risk factors in older women. Interantional Journal of Environmental Research and Public Health. 2020 Jun 5;17(11):4043. doi: 10.3390/ ijerph17114043.

[35] Mitchell RJ, Lord SR, Harvey LA, Close JC. Obesity and falls in older people: mediating effects of disease, sedentary behavior, mood, pain and medication use. Archives of Gerontology and Geriatrics. 2015 Jan-Feb;60(1):52-8. doi: 10.1016/j.archger.2014.09.006.

[36] Ellmers TJ, Cocks AJ, Young WR. Evidence of a link between fall-related anxiety and high-risk patterns of visual search in older adults during adaptive locomotion. Journal of Gerontology A Biological Sciences Medical Sciences. 2020;75(5):961-967. doi: 10.1093/gerona/ glz176.

[37] Viaje S, Crombez G, Lord SR, Close JCT, Sachdev P, Brodaty H, Delbaere K. The role of concern about falling on stepping performance during complex activities. BMC Geriatrics.2019 Nov 27;19(1):333. doi: 10.1186/s12877-019-1356-z.

[38] Choi NG, Bruce ML, DiNitto DM, Marti CN, Kunik ME. Fall worry restricts social engagement in older 
Falls Injuries and Anxiety among Community-Dwelling Older Adults in the Midst of COVID-19 Lockdown Restrictions: Are these Helpful or Harmful?

adults. Journal of Aging and Health. 2020;32(56):422-431. doi: 10.1177/0898264319825586..

[39] Zhao YL, Alderden J, Lind B, Stibrany J. Risk factors for falls in homebound communitydwelling older adults. Public Health Nursing. 2019;36(6):772-778. doi: 10.1111/phn.12651.

[40] De La Cámara MÁ, Jiménez-Fuente A, Pardos AI. Falls in older adults: the new pandemic in the post COVID-19 era?. Medical Hypotheses. 2020;145:110321. doi:10.1016/j. mehy.2020.110321

[41] Pelicioni PHS, Lord SR. COVID-19 will severely impact older people's lives, and in many more ways than you think! Brazilian Journal of Physical Therapy. 2020;24(4):293-294. doi: 10.1016/j. bjpt.2020.04.005.

[42] Harmon KJ, Fliss MD, Marshall SW, Peticolas K, Proescholdbell SK, Waller AE. The impact of the COVID-19 pandemic on the utilization of emergency department services for the treatment of injuries. American Journal of Emergency Medicine. 2021 Apr 20;47:187-191. doi: 10.1016/j.ajem.2021.04.019.

[43] Mukai R, Hasegawa S, Umetsu R, Nakao S, Shimada K, Uranishi H, Masuta M, Suzuki H, Nishibata Y, Nakamura M. Evaluation of pregabalininduced adverse events related to falls using the FDA adverse event reporting system and Japanese Adverse Drug Event Report databases. Journal of Clinical Pharmacy and Therapeutics. 2019;44(2):285-291. doi: 10.1111/jcpt.12790.

[44] Haddad YK, Karani MV, Bergen G, Marcum ZA. Willingness to change medications linked to increased fall risk: a comparison between age groups. Journal of the American Geriatric Society. 2019;67(3):527-533. doi: 10.1111/jgs.15696.

[45] Sitdhiraksa N, Piyamongkol P, Chaiyawat P, Chantanachai T, Ratta-Apha W, Sirikunchoat J, Pariwatcharakul P. Prevalence and factors associated with fear of falling in communitydwelling Thai elderly. Gerontology. 2021 Mar 5:1-5. doi: 10.1159/000512858.

[46] Karlsson LK, Jakobsen LH, Hollensberg L, Ryg J, Midttun M, Frederiksen H, Glenthøj A, Kodahl
AR, Secher-Johnsen J, Nielsen LK, Bofill NG, Knudtzen FC, Lund CM. Clinical presentation and mortality in hospitalized patients aged $80+$ years with COVID-19-A retrospective cohort study. Archives of Gerontology and Geriatics. 2021 May-Jun;94:104335. doi: 10.1016/j. archger.2020.104335..

[47] Downton JH, Andrews K. Prevalence, characteristics and factors associated with falls among the elderly living at home. Aging Clinical and Experimental Research. 1991;3(3):219-228.

[48] Iaboni A, Flint, AJ. The complex interplay of depression and falls in older adults: a clinical review. The American Journal of Geriatric Psychiatry. 2013;21(5):484-492.

[49] Zhao YL, Alderden J, Lind BK, Kim H. A comprehensive assessment of risk factors for falls in community-dwelling older adults. Journal of Gerontology and Nursing. 2018;44(10):40-48. doi: 10.3928/00989134-20180913-04.

[50] Al-Rahimi JS, Nass NM, Hassoubah SA, Wazqar DY, Alamoudi SA. Levels and predictors of fear and health anxiety during the current outbreak of COVID-19 in immunocompromised and chronic disease patients in Saudi Arabia: A cross-sectional correlational study. PLoS One. 2021;16(4):e0250554. doi: 10.1371/journal. pone.0250554.

[51] Gabell A, Simons MA, Nayak US. Falls in the healthy elderly: predisposing causes. Ergonomics. 1985;28(7):965-975.

[52] Eisenbeck N, Pérez-Escobar JA, Carreno DF. Meaning-centered coping in the era of covid-19: direct and moderating effects on depression, anxiety, and stress. Frontiers in Psychology. 2021 Mar 17;12:648383. doi: 10.3389/ fpsyg.2021.648383.

[53] Oliver-Welsh L, Richardson C, Ward DA. The hidden dangers of staying home: a London trauma unit experience of lockdown during the COVID-19 virus pandemic. Annals of the Royal College of Surgeons England. 2021;103(3):160166. doi: 10.1308/rcsann.2020.7066.

[54] Dunsky A. The effect of balance and coordination exercises on quality of life in older adults: a mini- 
Falls Injuries and Anxiety among Community-Dwelling Older Adults in the Midst of COVID-19 Lockdown Restrictions: Are these Helpful or Harmful?

review. Frontiers of Aging Neuroscience. 2019 Nov 15;11:318. doi: 10.3389/fnagi.2019.00318.

[55] WetherellJL, BowerES,Johnson K,Chang DG, Ward SR, Petkus AJ. Integrated exposure therapy and exercise reduces fear of falling and avoidance in older adults: a randomized pilot study. American Journal of Geriatric Psychiatry. 2018;26(8):849859. doi: 10.1016/j.jagp.2018.04.001.

[56] Pary R, Sarai SK, Micchelli A, Lippmann S. Anxiety disorders in older patients. Primary Care Companion CNS Disorders. 2019;21(1):18nr02335. doi: 10.4088/ PCC.18nr02335.

[57] GillTM, Bhasin S, Reuben DB, Latham NK, Araujo K, Ganz DA, Boult C, Wu AW, Magaziner J, Alexander N, Wallace RB, Miller ME, Travison TG, Greenspan SL, Gurwitz JH, Rich J, Volpi E, Waring SC, Manini TM, Min LC, Teresi J, Dykes PC, McMahon S, McGloin JM, Skokos EA, Charpentier P, Basaria S, Duncan PW, Storer TW, Gazarian P, Allore HG,
Dziura J, Esserman D, Carnie MB, Hanson C, Ko F, Resnick NM, Wiggins J, Lu C, Meng C, Goehring L, Fagan M, Correa-de-Araujo R, Casteel C, Peduzzi $P$, Greene EJ. Effect of a multifactorial fall injury prevention intervention on patient well-being: the STRIDE Study. Journal of the American Geriatric Society. 2021;69(1):173-179. doi: 10.1111/jgs.16854.

[58] Kaufman-Shriqui V, Navarro DA, Raz O, Boaz M. Dietary changes and anxiety during the coronavirus pandemic: a multinational survey. Europena Journal of Clinical Nutrition. 2021 Mar 19:1-9. doi: 10.1038/s41430-021-00897-3.

[59] Lim XY, Yap AC, Mahendran R, Yu J. The interplay between anxiety, fear, protective behaviors, compassion, and resilience among older adults during a COVID-19 lockdown: a structural equation modeling study. Translational Behavioral Medicine. 2021 Apr 1:ibaa143. doi: 10.1093/tbm/ibaa143.

Citation: Ray Marks. Falls Injuries and Anxiety among Community-Dwelling Older Adults in the Midst of COVID-19 Lockdown Restrictions: Are these Helpful or Harmful? . Open Journal of Geriatrics. 2021; 4(1): 14-25. DOI: https://doi.org/10.22259/2639-359X.0401002

Copyright: (C) 2021 Ray Marks. This is an open access article distributed under the Creative Commons Attribution License, which permits unrestricted use, distribution, and reproduction in any medium, provided the original work is properly cited. 